\title{
Salud y rendimiento académico en adolescentes Proyecto DADOS
}

\author{
Irene Monzonís Carda \\ imonzoni@uji.es \\ Mireia Adelantado Renau \\ adelantm@uji.es \\ Maria Reyes Beltran Valls \\ vallsm@uji.es \\ Carlos Bou Sospedra \\ carlosbousospedra@gmail.com \\ Diego Moliner Urdiales \\ dmoliner@uji.es
}

Life Research Group, Universitat Jaume I, Castellón

\section{Resumen}

Introducción: Los estudios que analizan el efecto combinado de diversos factores de salud sobre el rendimiento académico son escasos. El objetivo de este trabajo fue analizar el efecto combinado de cuatro factores relacionados con la salud (composición corporal, calidad de sueño, concentración de leptina y resistencia cardiorrespiratoria) sobre el rendimiento académico en adolescentes.

Métodos: La muestra estuvo formada por un total de 262 adolescentes $(13,9 \pm 0,3$ años), participantes del proyecto DADOS (Deporte, Adolescencia y Salud). La composición corporal se evaluó mediante el índice de masa corporal. La calidad de sueño se midió utilizando la versión española del cuestionario Pittsburgh Sleep Quality Index. La concentración de leptina se analizó en plasma empleando un kit ELISA. La resistencia cardiorrespiratoria se evaluó con el test de ida y vuelta de $20 \mathrm{~m}$. El rendimiento académico se evaluó mediante las calificaciones académicas.

Resultados: Los análisis de covarianza revelaron que los adolescentes con $\geq 3$ factores saludables mostraron un mejor rendimiento académico que aquellos con $\leq 1(p \leq 0,01)$. En términos de odds ratios $(\mathrm{OR})$, aquellos adolescentes que presentaban una composición corporal y una concentración de leptina adecuadas, buena calidad de sueño y niveles altos de resistencia cardiorrespiratoria mostraron mejores resultados en matemáticas $(\mathrm{OR}=6,02$; $95 \% \mathrm{Cl}=1,48-24,54)$, valenciano $(\mathrm{OR}=4,42 ; 95 \% \mathrm{Cl}=1,12-17,43)$ y en la 
calificación media $(\mathrm{OR}=8,68 ; 95 \% \quad \mathrm{Cl}=1,96-38,51)$ que el resto de sus compañeros.

Conclusión: Estos hallazgos revelan el efecto combinado de diversos factores de salud sobre el rendimiento académico en adolescentes. Los profesionales del ámbito sanitario y educativo deberían trabajar conjuntamente teniendo en cuenta esta relación.

Palabras clave: adolescencia, hábitos saludables, rendimiento aca démico, condición física, sueño.

\section{Abstract}

Introduction: Studies analysing the combined effect of healthy-related factors on academic performance in adolescents are scarce. Thus, the aim of the present study was to analyse the combined effect of four health-related factors (i.e. weight status, sleep quality, leptin concentration and cardiorespiratory fitness) on academic performance in adolescents.

Methods: A total of 262 adolescents $(13.9 \pm 0.3$ years) from the DADOS (Deporte, Adolescencia y Salud) study were included in the analyses. Weight status was assessed through body mass index. Sleep quality was measured using the Spanish version of the Pittsburgh Sleep Quality Index questionnaire. Plasma leptin concentration was measured using a sensitive ELISA kit. Cardiorespiratory fitness was assessed through the $20 \mathrm{~m}$ shuttle run test. Academic performance was assessed through school grades.

Results: Adolescents with at least 3 health-related factors showed better academic performance than those with 1 or zero factors $(p \leq 0.01)$. In terms of odds ratios (OR), adolescents with 4 health-related factors were more likely to be in the high-performance groups for Math (OR=6.02, 95\% Cl=1.48-24.54), Valencian $(\mathrm{OR}=4.42,95 \% \mathrm{Cl}=1.12-17.43)$ and grade point average $(\mathrm{OR}=8.68$, 95\% $\mathrm{Cl}=1.96-38.51)$.

Conclusions. These findings complement prior knowledge revealing the positive combined effect of several health-related factors on academic performance in adolescents. Health promotion initiatives and education programs should work together having in mind the relationship identified between health parameters and academical performance.

Keywords: adolescence, healthy lifestyles, academic performance, physical fitness, sleep.

\section{Introducción}

El rendimiento académico puede definirse como los objetivos educativos que deben alcanzar los estudiantes durante un determinado período de tiempo (Donnelly et al. 2016) y puede evaluarse a través de las notas o mediante cuestionarios estandarizados. Aunque el rendimiento académico parece estar fuertemente relacionado con las capacidades cognitivas, este también se relaciona con otras habilidades no cognitivas, como la motivación, el esfuerzo, la actitud, el interés, el apoyo familiar, la personalidad o la educación recibida (Petrides, Chamorro Premuzic, Frederickson y Furnham 2005). Un rendimiento académico alto durante la adolescencia se considera un predictor de salud y empleabilidad en la etapa adulta 
(French, Homer, Popovici y Robins 2015; Lê-Scherban, Diez Roux, Li y Morgenstern 2014), de ahí el interés que despierta el tema entre educadores y profesionales de la salud.

Estudios previos han analizado la influencia de diversos factores de salud sobre el rendimiento académico durante la infancia y la adolescencia, sugiriendo que estos factores no actúan de forma aislada, sino que podrían influ ir de forma conjunta sobre el rendimiento académico (Bradley y Greene 2013). Sin embargo, hasta la fecha, solamente tres estudios han examinado el efecto combinado de múltiples factores de salud sobre el rendimiento académico de niños y adolescentes, mostrando una relación positiva (Faught et al. 2017; Ickovics et al. 2014; Martínez Gómez et al. 2012).

Aunque investigaciones previas han analizado el efecto combinado de algunos factores de salud sobre determinados indicadores de rendimiento académico (Faught et al. 2017; Ickovics et al. 2014; Martínez Gómez et al. 2012), resultan necesarias nuevas investigaciones que incluyan nuevos factores de salud (i.e. biomarcadores sanguíneos), e indicadores globales de rendimiento académico (i.e. calificación media) para conocer con más precisión la relación entre estos parámetros. Por consiguiente, el principal objetivo del presente estudio fue analizar el efecto combinado de cuatro factores de salud (composición corporal, calidad de sueño, concentración de leptina en sangre y capacidad de resistencia cardiorrespiratoria) sobre el rendimiento académico (matemáticas, valenciano y la calificación media) en alumnado de $2 .^{\circ}$ curso de educación se cundaria obligatoria (ESO).

\section{Método}

\section{Participantes y diseño del estudio}

Los datos del presente estudio pertenecen a la evaluación inicial del proyecto longitudinal DADOS (Deporte, Adolescencia y Salud) llevada a cabo entre febrero y mayo del 2015. El proyecto DADOS tiene como objetivo principal analizar la influencia de la práctica deportiva sobre la salud, el rendimiento académico y el nivel de bien estar psicológico durante la adolescencia. Un total de 262 adolescentes, con datos válidos de composición corporal, calidad de sueño, concentración de leptina, resistencia cardiorrespiratoria y rendimiento académico fueron incluidos en los análisis. Los participantes fueron seleccionados en centros escolares y clubs deportivos de Castellón de la Plana y todos cumplían con los criterios generales de inclusión del proyecto DADOS (i.e. cursar $2 .^{\circ}$ de ESO, sin patologías crónicas, ni sometidos a tratamientos farmacológicos).

Todos los participantes recibieron una explicación detallada del protocolo de investigación y tanto ellos como sus padres o tutores legales firmaron un consentimiento informado. El proyecto DADOS se ha desarrollado siguiendo las recomendaciones éticas de la Declaración de Helsinki 1961 (revisión de Fortaleza 2013) y su protocolo de valoración ha sido aprobado por la Comisión Deontológica de la Universitat Jaume I de Castellón.

\section{Composición corporal}

La talla y el peso se midieron por duplicado siguiendo procedimientos estandarizados (Beltran Valls et al. 2017) y el valor medio de cada uno de ellos fue utilizado en los análisis. La talla fue medida con una precisión de $0,1 \mathrm{~cm}$ mediante un estadiómetro SECA 213 (Hamburgo, Alemania) con los participantes descalzos. El peso fue medido 
con una precisión de 0,1 kg mediante una báscula SECA 861 (Hamburgo, Alemania) con los participantes vistiendo ropa ligera. El índice de masa corporal (IMC) fue calculado con la fórmula peso/talla al cuadrado $\left(\mathrm{kg} / \mathrm{m}^{2}\right)$.

\section{Calidad de sueño}

La calidad de sueño se evaluó mediante el cuestionario Pittsburgh Sleep Quality Index (PSQI) que incluye 19 preguntas clasificadas en siete componentes relacionados con el sueño: calidad subjetiva, duración, latencia, eficiencia habitual, trastornos, uso de medicación y disfunción durante el día (Royuela Rico y Macías Fernández 1997). Los siete componentes se evalúan en una escala ascendente de 0 a 3 puntos, pudiendo estar la puntuación total comprendida de 0 a 21 (alfa de Cronbach=0,81).

\section{Concentración de leptina}

La concentración de leptina en plasma sanguíneo se cuantificó mediante la técnica ELISA (EIA-2395, Enzyme-Linked Immunosorbent Assay; DRG Instruments GmbH, Marburg, Alemania). La sensibilidad del ensayo de medición de la concentración de leptina fue de $0,7 \mathrm{ng} / \mathrm{mL}$, con un coeficiente de variación intraensayo e interensayo de $<10$ y $<15 \%$, respectivamente.

\section{Resistencia cardiorrespiratoria}

La resistencia cardiorrespiratoria se evaluó mediante el test de $20 \mathrm{~m}$ de ida y vuelta (Course-Navette). Este test consiste en ir y volver corriendo una distancia de $20 \mathrm{~m}$. Se usan señales de audio para indicar el ritmo que se debe seguir. La velocidad inicial es de $8,5 \mathrm{~km} / \mathrm{h}$, con un incremento de $0,5 \mathrm{~km} / \mathrm{h} / \mathrm{min}$. El número final de periodos completados (precisión de 0,5 periodos) fue utilizado en los análisis.

\section{Índice de estilo de vida saludable}

Se creó un índice de estilo de vida saludable comprendido entre 0 y 4 en base al número de factores de salud que acumulaba cada adolescente: 1) no padecer obesidad o sobrepeso fue considerado como composición corporal óptima (Cole, Bellizzi, Flegal y Dietz 2000); 2) presentar un valor del PSQI $\leq 5$ fue considerado buena calidad de sueño (Buysse, Reynolds, Monk, Berman y Kupfer 1989); 3) tener una concentración de leptina por debajo del percentil 50 específico según sexo fue considerado como nivel adecuado; y 4) tener unos niveles de resistencia cardiorrespiratoria iguales o superiores al percentil 75 específico según sexo fue considerado como elevado nivel de resistencia cardiorrespiratoria.

\section{Rendimiento académico}

El rendimiento académico fue evaluado mediante las calificaciones finales de $1 .{ }^{\circ} \mathrm{de}$ ESO, proporcionadas por las secretarías académicas de los centros educativos. Las calificaciones finales, en una escala de 0 a 10 puntos, fueron utilizadas para establecer tres indicadores de rendimiento académico según la literatura científica previa: matemáticas, valenciano y la calificación media de todas las asignaturas (geografía e historia, ciencias naturales, matemáticas, castellano, valenciano, inglés y educación física). Los participantes fueron clasificados en dos grupos: rendimiento académico 
alto ( $\geq$ percentil 50 en las calificaciones académicas) y rendimiento académico bajo (< percentil 50 en las calificaciones académicas).

\section{Covariables}

Las covariables que fueron incluidas en el presente estudio y recogidas mediante cuestionario fueron el sexo de los participantes, su nivel de desarrollo madurativo y el nivel socioeconómico de la unidad familiar.

El nivel de desarrollo madurativo fue autorreportado por los participantes a partir de la observación de gráficos estándares sobre las características sexuales primarias y secundarias según el protocolo establecido por Tanner y Whitehouse (1976). Se evaluó en una escala de 1 a 5 el nivel de desarrollo del vello púbico y de las mamas en chicas y del vello púbico y genitales en chicos. El valor más alto de estos dos componentes fue utilizado para establecer el nivel de desarrollo madurativo global.

El nivel socioeconómico fue evaluado utilizando la adaptación en español del cuestionario Family Affluence Scale (FAS) desarrollado originalmente por Currie et al. (2008).

\section{Análisis estadístico}

Las características de la muestra de estudio se presentan como medias \pm desviación estándar o porcentajes. La normalidad de las variables se comprobó utilizando procedimientos gráficos y estadísticos (i.e. test de Kolmogorov-Smirnov) y todas mostraron cumplir con los supuestos de normalidad. Las diferencias entre sexos fueron examinadas mediante la prueba $t$ de Student para muestras independientes en el caso de variables continuas y mediante la prueba de chi-cuadrado en el caso de variables categóricas. Los análisis previos mostraron que no existía interacción entre sexos, por lo que la muestra fue analizada conjuntamente.

Se realizaron análisis de covarianza (ANCOVA) para examinar las diferencias en el rendimiento académico en base al índice de estilo de vida saludable, ajustando por sexo, nivel de desarrollo madurativo y nivel socioeconómico. Además, se llevaron a cabo análisis de regresión logística para analizar la probabilidad de obtener un rendimiento académico alto en base al índice de estilo de vida saludable. Se realizaron dos modelos: modelo 1 (sin ajustar) y modelo 2 (ajustando por sexo, nivel de desarrollo madurativo y nivel socioeconómico).

\section{Resultados}

Las características de la muestra se presentan en la tabla 1. Comparados con las chicas, los chicos presentaban una mejor calidad de sueño $(4,2$ frente a 5,$5 ; p<0,001)$, una menor concentración de leptina en sangre $(1,6$ frente a 6,$1 ; p<0,001)$ y niveles más elevados de resistencia cardiorrespiratoria $(9,0$ frente a 6,$2 ; p<0,001)$. No se encontraron diferencias estadísticamente significativas en la composición corporal y en el rendimiento académico entre chicos y chicas. 
Tabla 1

Características de los participantes

\begin{tabular}{|c|c|c|c|c|}
\hline & Todos & Chicos & Chicas & $\mathbf{P}$ \\
\hline $\mathrm{n}(\%)$ & $262(100)$ & $137(52,3)$ & $125(47,7)$ & \\
\hline Edad (años) & $13,9 \pm 0,3$ & $13,9 \pm 0,3$ & $13,9 \pm 0,3$ & 0,956 \\
\hline Desarrollo madurativo (I-V) (\%) & 0/8/34/49/9 & $0 / 10 / 32 / 44 / 14$ & $0 / 6 / 35 / 54 / 5$ & - \\
\hline Nivel socioeconómico $(0-8)$ & $4,2 \pm 1,4$ & $4,0 \pm 1,3$ & $4,4 \pm 1,4$ & 0,031 \\
\hline \multicolumn{5}{|l|}{ Composición corporal } \\
\hline Altura $(\mathrm{cm})$ & $163,1 \pm 7,9$ & $164,7 \pm 8,6$ & $161,4 \pm 6,7$ & $<0,001$ \\
\hline Peso (kg) & $54,2 \pm 9,3$ & $54,5 \pm 9,7$ & $53,9 \pm 8,8$ & 0,574 \\
\hline Índice de masa corporal $\left(\mathrm{kg} / \mathrm{m}^{2}\right)$ & $20,3 \pm 2,7$ & $20,0 \pm 2,6$ & $20,6 \pm 2,9$ & 0,053 \\
\hline Sobrepeso u obesidad* $(\%)$ & 13,0 & 11,7 & 14,4 & 0,513 \\
\hline \multicolumn{5}{|l|}{ Calidad de sueño } \\
\hline Puntuación (0-21) & $4,8 \pm 2,8$ & $4,2 \pm 2,7$ & $5,5 \pm 2,7$ & $<0,001$ \\
\hline Buena calidad de sueño (\%) & 64,1 & 73,7 & 53,6 & $<0,001$ \\
\hline \multicolumn{5}{|l|}{ Leptina en sangre } \\
\hline Concentración (ng/mL) & $3,7 \pm 4,6$ & $1,6 \pm 2,8$ & $6,1 \pm 4,9$ & $<0,001$ \\
\hline Concentración adecuada (\%) & 51,5 & 48,9 & 48,0 & 0,884 \\
\hline \multicolumn{5}{|l|}{ Resistencia cardiorrespiratoria } \\
\hline Periodos completados & $7,7 \pm 2,5$ & $9,0 \pm 2,0$ & $6,2 \pm 2,2$ & $<0,001$ \\
\hline Niveles elevados (\%) & 29,0 & 25,5 & 32,8 & 0,196 \\
\hline $\begin{array}{l}\text { Índice de estilo de vida saludable } \\
(0-4)(\%)\end{array}$ & $5 / 21 / 32 / 25 / 17$ & $3 / 18 / 36 / 26 / 17$ & $6 / 24 / 29 / 25 / 16$ & - \\
\hline \multicolumn{5}{|l|}{ Rendimiento académico $(0-10)$} \\
\hline Matemáticas & $6,9 \pm 1,6$ & $7,0 \pm 1,6$ & $6,8 \pm 1,6$ & 0,316 \\
\hline Valenciano & $6,8 \pm 1,5$ & $6,6 \pm 1,5$ & $6,9 \pm 1,5$ & 0,123 \\
\hline Calificación media & $7,1 \pm 1,3$ & $7,0 \pm 1,3$ & $7,2 \pm 1,3$ & 0,306 \\
\hline
\end{tabular}

Los datos se presentan como media \pm desviación estándar o porcentajes. Las diferencias entre el sexo fueron examinadas a travprueba $t$ de Student o chi-cuadrado. Los valores estadísticamente significativos se muestran en negrita.

Los análisis de covarianza (ANCOVA) examinando las diferencias en el rendimiento académico en base al índice de estilo de vida saludable, ajustando por sexo, nivel de desarrollo madurativo y nivel socioeconómico, se presentan en la figura 1. Los adolescentes que presentaban un índice de estilo de vida saludable de 4 factores mostraron un mejor rendimiento académico en matemáticas comparado con aquellos con un índice de uno o cero factores $(p \leq 0,01)$. Además, aquellos adolescentes con un índice de estilo de vida saludable de 3 o más factores presentaron un mejor rendimiento académico en la calificación media que aquellos con uno o cero factores $(p \leq 0,01)$. 

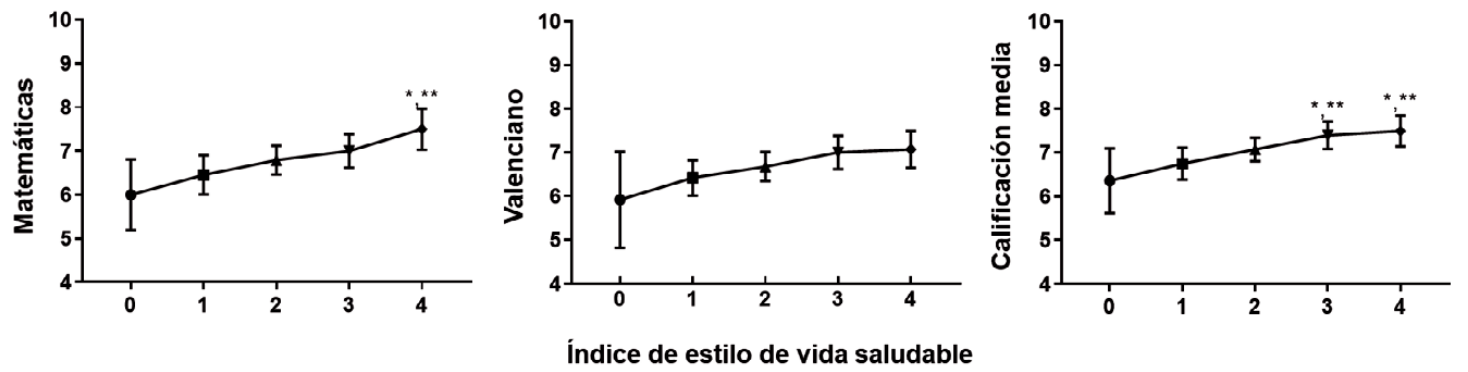

Figura 1. Efecto combinado de la composición corporal, la calidad de sueño, la concentración de leptina y la resistencia cardiorrespiratoria sobre el rendimiento académico. Las medias (puntos) y el IC $95 \%$ (intervalo de confianza, barras de error) representan los valores después de ajustar por sexo, nivel de desarrollo madurativo y nivel socioeconómico $(n=262)$.

*indica diferencias significativas con el primer grupo (índice de estilo de vida saludable $=0$ ); **indica diferencias significativas con el segundo grupo (índice de estilo de vida saludable=1); $p<0,05$.

Los análisis de regresión logística para analizar la probabilidad de obtener un rendimiento académico alto en base al índice de estilo de vida saludable se muestran en la tabla 2. Los adolescentes con un índice de estilo de vida saludable de 4 mostraron mayor probabilidad de obtener un alto rendimiento académico en matemáticas $(\mathrm{OR}=5,33 ; \quad 95 \% \quad \mathrm{Cl}=1,35-21,02), \quad$ valenciano $\quad(\mathrm{OR}=3,87 ; \quad 95 \%$ $\mathrm{Cl}=1,00-14,95)$ y en la calificación media $(\mathrm{OR}=7,15 ; 95 \% \mathrm{Cl}=1,66-30,75)$, comparado con sus compañeros con un índice de 0 (modelo 1 ). Tras ajustar por sexo, nivel de desarrollo madurativo y nivel socioeconómico (modelo 2 ), aquellos adolescentes que presentaban un índice de estilo de vida saludable de 4 mostraron mayor probabilidad de obtener un alto rendimiento académico en matemáticas (OR=6,02; $95 \% \mathrm{Cl}=1,48$ $24,54)$, en valenciano $(\mathrm{OR}=4,42 ; 95 \% \mathrm{Cl}=1,12-17,43)$ y en la calificación media $(\mathrm{OR}=8,68 ; 95 \% \mathrm{Cl}=1,96-38,51)$; y los que presentaron un índice de estilo de vida de 3 mostraron un mayor rendimiento académico en la calificación media $(\mathrm{OR}=4,63 ; 95 \%$ $\mathrm{Cl}=1,12-19,21)$.

Tabla 2

Análisis de regresión logística examinando el efecto combinado de diversos factores de salud sobre el rendimiento académico en adolescentes $(n=262)$

\begin{tabular}{|c|c|c|c|c|c|c|}
\hline \multirow{2}{*}{$\begin{array}{l}\text { Índice } \\
\text { estilo } \\
\text { de vida }\end{array}$} & \multicolumn{2}{|c|}{ Matemáticas } & \multicolumn{2}{|c|}{ Valenciano } & \multicolumn{2}{|c|}{ Calificación media } \\
\hline & Modelo 1 & Modelo 2 & Modelo 1 & Modelo 2 & Modelo 1 & Modelo 2 \\
\hline \multicolumn{7}{|l|}{ (a) } \\
\hline 0 & 1,00 (referencia) & 1,00 (referencia) & 1,00 (referencia) & 1,00 (referencia) & 1,00 (referencia) & 1,00 (referencia) \\
\hline 1 & $1,33(0,36-4,97)$ & $1,40(0,37-5,38)$ & $1,24(0,33-4,61)$ & $1,36(0,36-5,15)$ & $2,32(0,57-9,52)$ & $2,63(0,63-11,03)$ \\
\hline 2 & $2,15(0,60-7,67)$ & $2,45(0,66-9,04)$ & $2,60(0,73-9,28)$ & $3,06(0,84-11,17)$ & $2,80(0,71-11,05)$ & $3,48(0,85-14,19)$ \\
\hline 3 & $2,55(0,70-9,32)$ & $2,86(0,76-10,77)$ & $2,40(0,66-8,76)$ & $2,76(0,74-10,24)$ & $3,83(0,95-15,43)$ & $4,63(1,12-19,21)$ \\
\hline 4 & $5,33(1,35-21,02)$ & $6,02(1,48-24,54)$ & $3,87(1,00-14,95)$ & $4,42(1,12-17,43)$ & $7,15(1,66-30,75)$ & $8,68(1,96-38,51)$ \\
\hline
\end{tabular}

Los odds ratio (intervalo de confianza del $95 \%$ ) representan la probabilidad de conseguir un rendimiento académico alto. Modelo 1: no ajustado; modelo 2: ajustado por sexo, nivel de desarrollo madurativo y nivel socioeconómico. Los resultados significativos se muestran en negrita. 


\section{Discusión y conclusiones}

Los resultados principales de este estudio sugieren que los alumnos de $2 .^{\circ}$ de ESO con una composición corporal adecuada, buena calidad de sueño, niveles adecuados en la concentración de leptina y niveles elevados de resistencia cardiorrespiratoria mostraron una mayor probabilidad de presentar un rendimiento académico alto. Estos hallazgos complementan los estudios previos indicando una asociación positiva y combinada de diversos factores de salud sobre el rendimiento académico en adolescentes.

Nuestros resultados coinciden con estudios previos que ponen de manifiesto una relación positiva e independiente entre la composición corporal saludable (Larsen, Kleinjan, Engels, Fisher y Hermans 2014), la óptima calidad de sueño (Dewald, Meijer, Oort, Kerkhof y Bogels 2010), la adecuada concentración de leptina (Correa Burrows et al. 2016) y la elevada capacidad cardiorrespiratoria (Marques, Santos, Hillman y Sardinha 2017) con el rendimiento académico en adolescentes. Algunos estudios sugieren que estos factores no actúan de forma aislada y que su efecto combinado sobre el rendimiento académico es incluso mayor que el de cada factor de forma aislada (Faught et al. 2017). El efecto combinado y positivo encontrado en nuestro estudio coincide con los resultados obtenidos en investigaciones previas (Faught et al. 2017; Ickovics et al. 2014; Martínez Gómez et al. 2012). Por ejemplo, Martínez Gómez et al. (2012) analizaron una muestra de 1825 adolescentes españoles mostrando que las chicas que reunían 3 o 4 factores de comportamiento saludable, incluyendo actividad física, tiempo de pantalla, duración del sueño y el consumo de frutas, presentaban entre 1,75 y 3,18 veces más probabilidad de obtener mejores resultados en matemáticas y lengua, respectivamente, comparadas con aquellas que reunían $\leq 1$ factor saludable. De forma similar, otro estudio realizado en Estados Unidos analizó 14 factores de salud (incluyendo composición corporal, condición física, dieta, actividad física, tiempo de pantalla, fumar, bienestar y calidad de sueño) concluyendo que aquellos niños que seguían las recomendaciones saludables eran más propensos a alcanzar los objetivos en matemáticas, escritura y lectura (Ickovics et al. 2014). Por último, en un estudio más reciente, Faught et al. (2017) reportaron que aquellos adolescentes que cumplían entre 7 y 9 recomendaciones saludables (incluyendo recomendaciones de actividad física, tiempo de pantalla, duración del sueño y patrones saludables de dieta) presentaban mejores resultados en matemáticas, lectura y escritura.

Los que subyacen tras esta asociación positiva entre factores de salud y rendimiento académico quedan fuera del objeto del presente estudio. No obstante, especulamos que los resultados obtenidos podrían deberse al efecto acumulado de cada uno de los factores de salud sobre el rendimiento académico. De hecho, el efecto combinado de los factores saludables incluidos en este estudio podría mejorar la función cognitiva (i.e. inhibición, memoria) y favorecer el bienestar psicológico de los adolescentes (Dewald, Meijer, Oort, Kerkhof y Bogels 2010; Gunnarsdottir, Njardvik, Olafsdottir, Craighead y Bjarnason 2012), lo que a su vez conllevaría una mejora en el rendimiento académico. Además, el hecho de mantener un estado de salud óptimo podría reducir el absentismo escolar (Pan, Sherry, Park y Blanck 2013) así como el cansancio (Dewald, Meijer, Oort, Kerkhof y Bogels 2010), lo que se podría reflejar en un mayor rendimiento académico.

Conseguir un óptimo rendimiento académico durante la etapa de educación obligatoria constituye una preocupación para las familias y los profesionales implicados, tanto por sus consecuencias positivas sobre la salud como sobre el desarrollo laboral en la etapa adulta, con los consecuentes beneficios socioeconómicos (French, Homer, Popovici y Robins 2015; Lê-Scherban, Diez Roux, Li y Morgenstern 2014). Teniendo 
en cuenta nuestros resultados, parece clave la promoción de óptimos estándares de salud en el ámbito educativo, pues de forma conjunta contribuyen a la mejora del rendimiento académico. Además, los hallazgos de nuestro estudio resaltan la importancia de un enfoque multidisciplinar al diseñar programas para la mejora del rendimiento académico en adolescentes.

\section{Limitaciones y puntos fuertes}

Nuestros resultados deben ser interpretados con cautela debido al diseño transversal del estudio, que no permite establecer relaciones causa-efecto. No obstante, este estudio presenta diversos puntos fuertes entre los que cabe destacar la homogeneidad de los participantes en cuanto a su edad, el uso de cuestionarios y test de campo validados, la inclusión de parámetros sanguíneos y el control de la influencia de variables de gran relevancia para la salud y el rendimiento académico, como son el grado de desarrollo madurativo y el nivel socioeconómico de los participantes.

\section{Conclusiones}

Los resultados del presente estudio revelan un efecto combinado y positivo de diversos factores de salud sobre el rendimiento académico en adolescentes. Las iniciativas de promoción de la salud en educación deberían considerar estos factores de manera conjunta, en vez de centrarse en ellos individualmente. Los profesionales de la educación y de la salud deberían trabajar conjuntamente para mejorar tanto el estado de salud como el rendimiento académico de los adolescentes. Asimismo, se requieren futuros estudios que clarifiquen la relación entre diversos factores de salud sobre el rendimiento académico y cognitivo de los adolescentes.

\section{Referencias bibliográficas}

Beltran Valls, Maria Reyes, Enrique García Artero, Ana Capdevila Seder, Alejandro Legaz Arrese, Mireia Adelantado Renau y Diego Moliner Urdiales. 2017. «Regular Practice of Competitive Sports Does Not Impair Sleep in Adolescents: DADOS Study». Pediatric exercise science, 30(2): 229-236.

Bradley, Beverly J. y Amy C. Greene. 2013. "Do health and education agencies in the United States share responsibility for academic achievement and health? A review of 25 years of evidence about the relationship of adolescents' academic achievement and health behaviors». Journal of adolescent health, 52(5): 523-532.

Buysse, Daniel J., Charles F. Reynolds, Timothy H. Monk, Susan R. Berman y David J. Kupfer. 1989. "The Pittsburgh sleep quality index: A new instrument for psychiatric practice and research». Psychiatry research, 28(2): 193-213.

Cole, Tim J., Mary C. Bellizzi, Katherine M. Flegal y William H. Dietz. 2000. "Establishing a standard definition for child overweight and obesity worldwide: international survey». BMJ (Clinical research Ed.), 320(7244): 1240-1243.

Correa Burrows, Paulina, Estela Blanco, Marcela Reyes, Marcela Castillo, Patricio Peirano, Cecilia Algarín, Betsy Lozoff, Sheila Gahagan y Raquel Burrows. 2016. "Leptin status in adolescence is associated with academic performance in high school: a cross-sectional study in a Chilean birth cohort». BMJ Open, 6(10): e010972.

Currie, Candace, Michal Molcho, William Boyce, Bjørn Holstein, Torbjørn Torsheim y Matthias Richter. 2008. «Researching health inequalities in adolescents\%: The 
development of the Health Behaviour in School-Aged Children (HBSC) Family Affluence Scale». Social Science \& Medicine, 66: 1429e1436.

Dewald, Julia F., Anne M. Meijer, Frans J. Oort, Gerard A. Kerkhof y Susan M. Bogels. 2010. "The influence of sleep quality, sleep duration and sleepiness on school performance in children and adolescents: A meta-analytic review». Sleep medicine reviews, 14(3): 179-189.

Donnelly, Joseph E., Charles H. Hillman, Darla M. Castelli, Jennifer L. Etnier, Sarah Lee, Phillip Tomporowski, Kate Lambourne y Amanda N. Szabo-reed. 2016. «Physical Activity, Fitness, Cognitive Function, and Academic Achievement in Children: A Systematic Review». Medicine and science in sports and exercise, 48(6), 1197-1222.

Faught, Erin L., John P. Ekwaru, Douglas Gleddie, Kate E. Storey, Mark Asbridge y Paul J. Veugelers. 2017. "The combined impact of diet, physical activity, sleep and screen time on academic achievement: a prospective study of elementary school students in Nova Scotia, Canada». The international journal of behavioral nutrition and physical activity, 14(1): 29.

French, Michael T., Jenny F. Homer, Ioana Popovici y Philip K. Robins. 2015. «What You Do in High School Matters: High School GPA, Educational Attainment, and Labor Market Earnings as a Young Adult». Eastern economic journal, 41(3): 370-386.

Gunnarsdottir, Thrudur, Urdur Njardvik, Anna S. Olafsdottir, Linda W. Craighead y Ragnar Bjarnason. 2012. "Teasing and social rejection among obese children enrolling in family-based behavioural treatment: effects on psychological adjustment and academic competencies». International journal of obesity, 36(1): 35-44.

Ickovics, Jannette R., Amy Carrol Scott, Susan M. Peters, Marlene Schwartz, Kathryn Gilstad Hayden y Catherine McCaslin. 2014. "Health and Academic achievement: cumulative effects of health assets on standardized test scores among urban youth in the United States». Journal of school health, 84(1): 4048.

Larsen, Junilla K., Marloes Kleinjan, Rutger C. M. E. Engels, Jennifer O. Fisher y Roel Hermans. 2014. "Higher Weight, Lower Education: A Longitudinal Association Between Adolescents' Body Mass Index and Their Subsequent Educational Achievement Level?». Journal of school health, 84(12): 769-776.

Lê-Scherban, Félice, Ana V. Diez Roux, Yun Li y Hal Morgenstern. 2014. «Does academic achievement during childhood and adolescence benefit later health?». Annals of epidemiology, 24(5): 344-355.

Marques, Adilson, Diana A. Santos, Charles H. Hillman y Luís B. Sardinha. 2017. "How does academic achievement relate to cardiorespiratory fitness, selfreported physical activity and objectively reported physical activity: a systematic review in children and adolescents aged 6-18 years». British journal of sports medicine, 52(16): 1-11.

Martínez Gómez, David, Óscar L. Veiga, Sonia Gómez Martínez, Belén Zapatera, David Martínez Hernández, María E. Calle y Ascensión Marcos. 2012. "Gender-specific influence of health behaviors on academic performance in Spanish adolescents: the AFINOS study». Nutrición hospitalaria, 27(3): 724730.

Pan, Liping, Bettylou Sherry, Sohyun Park y Heidi M. Blanck. 2013. "The association of obesity and school absenteeism attributed to illness or injury among adolescents in the United States, 2009». The journal of adolescent health: official publication of the society for adolescent medicine, 52(1): 64-69.

Petrides, Konstantinos V., Tomas Chamorro Premuzic, Norah Frederickson y Adrian Furnham. 2005. «Explaining individual differences in scholastic behaviour and achievement». British journal of educational psychology, 75(2): 239-255. 
Royuela Rico, Angel y Jose A. Macías Fernández. 1997. «Propiedades Clinimétricas De La Versión Castellana Del Cuestionario De Pittsburg». Vigilia-Sueño, 9(2): 81-94.

Tanner, James M. y Reg H. Whitehouse. 1976. "Clinical longitudinal standards for height, weight, height velocity, weight velocity, and stages of puberty». Archives of disease in childhood, 51(3): 170-179. 